Urol. Prax. 2020 $22: 100-104$

https://doi.org/10.1007/s41973-020-00104-z

Online publiziert: 6 . August 2020

(c) Der/die Autor(en) 2020

\section{Wetterauer ${ }^{1} \cdot$ Helge Seifert ${ }^{1} \cdot$ A. Chirindel $\left.\right|^{2} \cdot$ G. Nicolas ${ }^{2}$}

' Klinik für Urologie, Universitätsspital Basel, Basel, Schweiz

${ }^{2}$ Klinik für Radiologie und Nuklearmedizin, Universitätsspital Basel, Basel, Schweiz

\title{
Die Rolle der PSMA-PET-CT in der Diagnostik und Therapie des Prostatakarzinoms
}

\section{Grundlagen der PSMA- Diagnostik}

In der Schweiz ist Prostatakrebs die häufigste Krebserkrankung bei Männern [1] und die zweithäufigste tumorassoziierte Todesursache [2]. Zwischen 2012 und 2016 gab es jährlich $6089(115,7 / 100.000)$ Neuerkrankungen. Viele dieser Patienten entwickeln nach einer initial kurativen Therapie im weiteren Verlauf ein biochemisches Rezidiv. Die Tumorsuche mit konventionellen Methoden ist hierbei eine grosse Herausforderung. Über die letzten Jahre hat sich die PSMA-PET-CT (Prostataspezifisches-MembranantigenPositronenemissionstomographie-Computertomographie) als Goldstandard in der Rezidivsituation etabliert.

Grundlage der PET ist das nichtinvasive Sichtbarmachen molekularer oder physiologischer Prozesse in vivo mittels radioaktiv markierten Tracern (Radiopharmaka). PET-Radiopharmaka sind Substanzen, die mit einem $\beta^{+}$-Strahler als Radionuklid markiert sind, z. B. das radioaktive Isotop ${ }^{18} \mathrm{~F}$ des Fluors (Zyklotron-Produkt) und ${ }^{68} \mathrm{Ga}$ des Galliums (Generator-Produkt). Noch bis vor wenigen Jahren kam bei der PETCT-Diagnostik von Prostatakarzinomen ${ }^{18}$ Fluor $\left({ }^{18} \mathrm{~F}\right)$-markiertes Cholin zur Anwendung. Allerdings zeigte dieses Verfahren vor allem bei niedrigen PSAWerten (prostataspezifisches Antigen) und einem höheren Gleason-Score als Ausdruck einer fortgeschrittenen Entdifferenzierung, eine geringe Sensitivität und Spezifität. Als alternative Tracer wurden mit ${ }^{11} \mathrm{C}$ markiertes Acetat sowie ${ }^{18} \mathrm{~F}$-markiertes Fluciclovin klinisch erprobt, zeigten allerdings keine überlegenen Ergebnisse. Weitere vielversprechende Alternativen wie radiomarkierte Liganden des Gastrin-related-PeptideRezeptors (mit ${ }^{68} \mathrm{Ga}$-markiertem Bombesin-Antagonisten) werden aktuell im Rahmen klinischer Studien untersucht [3]. Der erste PSMA-PET-Ligand wurde 2002 von Pomper et al. von der Johns Hopkins University, Baltimore, USA, publiziert [4]. Seitdem wurden mehrere sowohl ${ }^{18} \mathrm{~F}$ - als auch ${ }^{68} \mathrm{Ga}$-markierte PSMA-Liganden entwickelt. Im Jahr 2011 gelang es am deutschen Krebsforschungszentrum in Heidelberg, den Liganden PSMA-11 zu entwickeln. Nach Kopplung an das radioaktive Metall ${ }^{68} \mathrm{Ga}$ konnte PSMA-11 erstmalig in der PETCT-Diagnostik eingesetzt werden.

Auf der Suche nach diagnostischen Alternativen ist die PSMA-basierte Bildgebung zunehmend in den Fokus der wissenschaftlichen Aufmerksamkeit gerückt. Hierbei hat sich PSMA als das bislang am besten geeignete tumorspezifische Zielmolekül in der Zellmembran für die molekulare Bildgebung herausgestellt. Dabei handelt es sich um ein transmembranes Enzym, von dem vermutet wird, dass es an angiogenetischen Prozessen beteiligt ist [5]. PSMA wird auf Prostatakrebszellen signifikant höher exprimiert als auf gesunden Zellen. Zudem steigt die PSMA-Expression auf schlecht differenzierten und/oder kastrationsrefraktären Zellen weiter an - und das unabhängig vom Metabolismus der Zelle [6].

Allerdings gilt es auch zu beachten, dass bis zu zehn Prozent der primären Prostatatumoren keine PSMA-
Expression aufweisen [7, 8]. In typischen Lokalisationen einer Prostatakarzinommetastasierung, wie Lymphknoten und Knochen, ist so gut wie keine physiologische PSMA-Expression zu verzeichnen, sodass Prostatakarzinomherde durch die PSMA-basierte Bildgebung mit ${ }^{68} \mathrm{Ga}$-PSMA-11 mit exzellentem Kontrast dargestellt werden können ([9]; - Abb. 1). Eine hohe physiologische Traceranreicherung findet man hingegen im Dünndarm, den Nieren sowie den Speichel- und Tränendrüsen. Aktuell werden sowohl ${ }^{18} \mathrm{~F}$ - als auch ${ }^{68} \mathrm{Ga}$-markierte PSMA-Liganden verwendet. ${ }^{68} \mathrm{Ga}-$ PSMA-11 und ${ }^{18}$ F-PSMA-1007 sind in der Schweiz seit 2016 resp. 2019 als PETRadiopharmaka zugelassen. Ein weiterer vielversprechender PSMA-PET-Ligand $\left({ }^{18} \mathrm{~F}\right.$-DCFPyl) wurde in einer PhaseIII-Studie (CONDOR) in Nordamerika vorgestellt, die positive Resultate bei Patienten mit biochemischem Rezidiv und negativer konventioneller Bildgebung (inkl. ${ }^{18} \mathrm{~F}$-Fluciclovin PET, Cholin PET, CT, MR und Knochenszintigraphie) zeigte (ASCO 2020).

\section{Hybridbildgebung}

Die simultane Bildgebung mittels PSMAPET-CT kombiniert die Vorteile der nuklearmedizinischen mit denen der radiologischen Bildgebung. Das GanzkörperPSMA-PET-CT erlaubt eine zuverlässige Aussage über die Ausbreitung der Erkrankung in der Prostataloge und den lokoregionären Lymphknoten sowie über das Vorliegen von Fernmetastasen. Weitere Vorteile der PSMA-PET-CT sind die steile Lernkurve sowie die geringe In- 

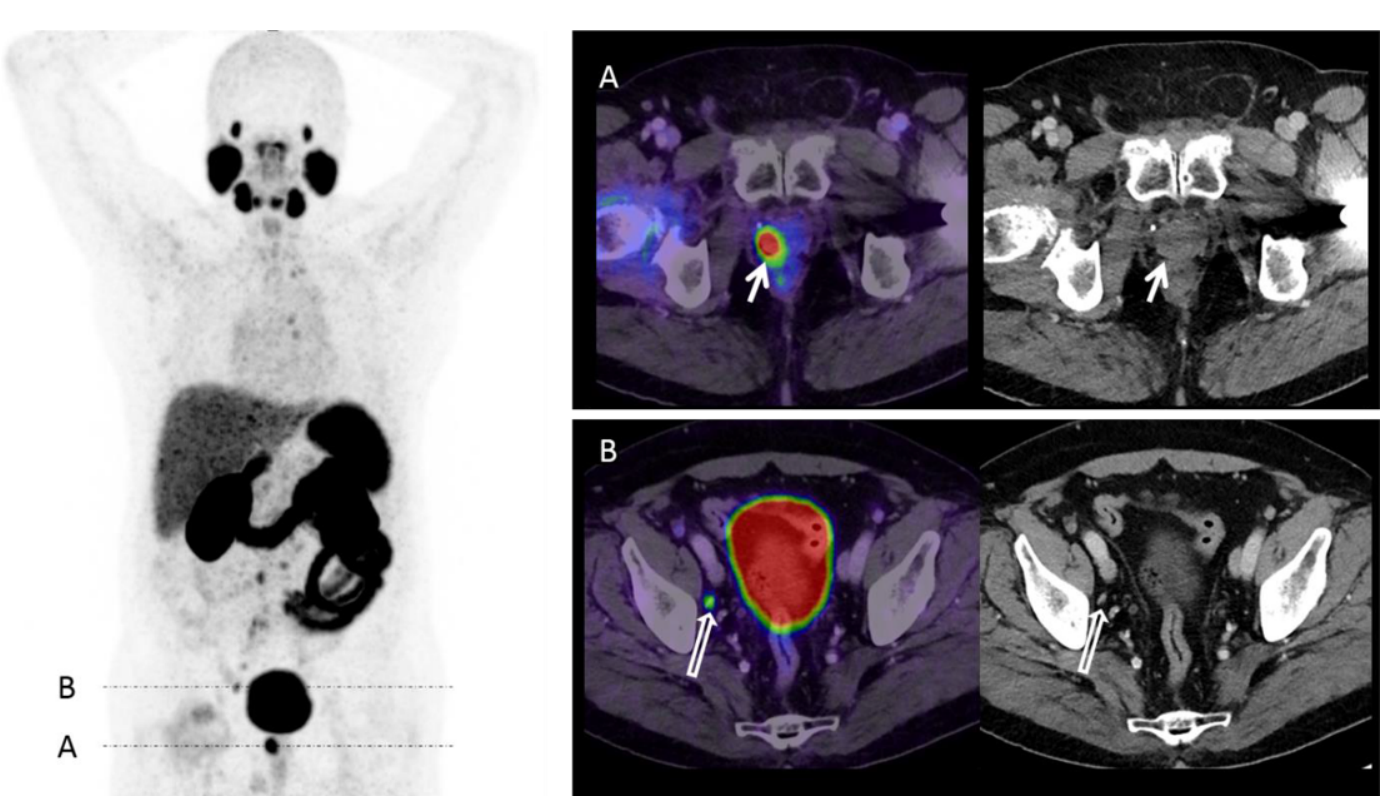

Abb. $1 \Delta{ }^{68}$ Ga-PSMA11-PET-CT mit PSMA-exprimierender Läsion in der Prostataloge $(A)$, pelvin $(B)$, retroperitoneal und mediastinal dringend verdächtig auf Lokalrezidiv und Lymphknotenmetastasen (exemplarisch rechts iliakal, $B$ ) bei biochemischem Rezidiv eines Prostatakarzinoms (initial cT3, Gleason 9) nach kurativ intendierter Radiotherapie und antihormoneller Therapie. Auf der mitgefahrenen kontrastmittelverstärkten CT (rechteSpalte) können die Befunde bildmorphologisch nicht als pathologisch erkannt werden. Ansonsten zeigt sich eine physiologische ${ }^{68} \mathrm{Ga}$-PSMA11-Speicherung in den Tränen- und Speicheldrüsen, der Milz, dem Duodenum und Jejunum sowie den Nieren und der Harnblase

terobservervariabilität bei der Beurteilung [9]. Die folgenden Abschnitte sollen einen Überblick über die Anwendung der PSMA-PET-CT in der Rezidivdiagnostik, der Primärdiagnostik und der Therapie metastasierter kastrationsresistenter Tumoren geben.

\section{PSMA-PET-CT in der Rezidivdiagnostik}

Nach initialer kurativer Therapie - durch radikale Prostatektomie oder Radiatio entwickeln 15-40\% aller Patienten ein biochemisches Rezidiv [10]. In dieser Situation stellt sich die Frage, ab welchem PSA-Wert eine PET-Untersuchung sinnvoll ist. Erwartungsgemäss steigt mit der Höhe des PSA-Werts auch die Wahrscheinlichkeit, einen Prostatakarzinomherd in der PSMA-PET-CT nachzuweisen [11]. Diese wurde in mehrere Arbeiten sowie in einer kürzlich publizierten Metaanalyse mit 1309 PSMA-PETCT-Untersuchungen nachgewiesen [12, 13]. Die PSMA-PET-CT zeigte bei einem PSA-Wert von $0,2 \mathrm{ng} / \mathrm{ml}$ in etwa $48 \%$ der Fälle positive Befunde. Die Detektionswahrscheinlichkeit stieg bei PSA-Werten von $0,5 \mathrm{ng} / \mathrm{ml}$ auf $56 \%$ und bei Werten von $1 \mathrm{ng} / \mathrm{ml}$ auf $70 \%$ [12]. Zudem konnte, wenn auch statistisch weniger valide, eine erhöhte Detektionswahrscheinlichkeit bei einer PSA-Verdopplungszeit von $<6$ Monaten im Vergleich zu einer PSA-Verdopplungszeit von $>6$ Monaten nachgewiesen werden. Mehrere Studien konnten bestätigen, dass die Spezifität der PSMA-PET-CT bei $>95 \%$ liegt [14, 15]. Dennoch muss betont werden, dass PSMA nicht organspezifisch ist und eine Expression auch von anderen neovaskularisierten Tumoren und benignem Gewebe beschrieben ist [16]. Nichtsdestotrotz muss bis zum Beweis des $\mathrm{Ge}$ genteils bei positiven PSMA-PET-CTBefunden von einer Metastasierung ausgegangen werden und bei unklaren Fällen eine bioptische Sicherung erfolgen. Aktuelle Daten belegen auch, dass eine Langzeit-ADT (antiandrogene Therapie) einen signifikant negativen Einfluss auf die Sichtbarkeit und damit die Detektion von kastrationssensitiven Tumorherden in der PSMA-PET-CT haben kann [17], bei kastrationsrefraktären Tumoren allerdings wurde ein positiver Einfluss nachgewiesen [18]. Die aktuellen EAU Guidelines für Urologie empfehlen die Durchführung einer PSMA-PET-CT in der Rezidivsituation nach Radiotherapie, wenn Patienten für eine kurative „Salvage-Therapie" geeignet sind oder nach radikaler Prostatektomie ab einem PSAWert $>0,2 \mathrm{ng} / \mathrm{ml}$, wenn die Ergebnisse die Therapieentscheidung beeinflussen[19]. Unabhängig von der diagnostischen $\mathrm{Ge}$ nauigkeit und dem Wunsch des behandelnden Arztes sowie des Patienten zu wissen, wie ein erhöhter PSA-Wert zustande kommt, ist die entscheidende Frage, ob eine PSMA-PET-CT dem Patienten zu einem therapeutischen Vorteil verhilft oder nicht, noch nicht abschliessend geklärt.

\section{PSMA-PET-CT in der Primärdiagnostik}

Für die Primärdiagnostik bei Prostatatumoren mit intermediärem oder hohem Risiko empfehlen die aktuellen EAU Guidelines für Urologie die Durchführung einer Computertomographie (CT) oder einer MRT des Abdomens in Kombination mit einer Knochenszintigraphie. Der Einsatz der PSMA-PET-CT für diese Indikation ist Gegenstand aktueller Untersuchungen. In einer multizentrisch randomisierten Studie mit 302 Pros- 
tatakrebspatienten mit hohem Risiko konnte die PSMA-PET-CT die diagnostische Genauigkeit für die Detektion von pelvinen Lymphknotenmetastasen im Vergleich zur konventionellen Bildgebung (CT und Knochenszintigraphie) von $59 \%$ auf $91 \%$ deutlich verbessern. Die Sensitivität und Spezifität für die PSMA-PET-CT lagen bei 85\% [74-96] und 91\% [85-97] und bei der konventionellen Bildgebung bei 38\% [24-52] und $98 \%$ [95-100] [20]. Gemäss den Ergebnissen einer kürzlich publizierten Studie scheint der Nachweis von kleinen Lymphknotenmetastasen um $4 \mathrm{~mm}$ mittels PSMA-PET-CT allerdings nicht möglich zu sein [21]. Diese Ergebnisse zeigen, dass trotz negativer PSMA-PET-CT eine Lymphknotenmetastasierung vorliegen kann. Hinsichtlich der Detektion von Fernmetastasen sind die Ergebnisse der PSMA-PET$\mathrm{CT}$ vielversprechend. Die diagnostische Genauigkeit der PSMA-PET-CT für die Detektion von Fernmetastasen lagen in der Studie von Hofman et al. bei $95 \%$ [20] im Vergleich zu 74\% bei Durchführung einer Knochenszintigraphie in Kombination mit einer CT [22].

Zudem zeichnet sich die PSMAPET-CT durch eine geringere Strahlenbelastung als die Kombination aus Knochenszintigraphie und CT aus. Diese Ergebnisse verdeutlichen das Potenzial der PSMA-PET-CT, die Primärdiagnostik zu verbessern und die konventionelle Bildgebung langfristig zu ersetzen und damit das Patientenmanagement nachhaltig zu verbessern. Es bedarf aber weiterer prospektiver Studien, die den Einfluss der PSMA-PET-CT auf das onkologische Outcome untersuchen.

\section{PSMA-gerichtete Radionuklid- therapie}

Analog zu den molekularen Mechanismen der PSMA-basierten Bildgebung kann auch eine PSMA-basierte Therapie erfolgen. Hierbei werden therapeutische Betastrahler wie ${ }^{177}$ Lutetium an den Liganden gekoppelt und können so ihre therapeutische Wirkung direkt an den Tumorzellen entfalten. Bei nur kurzer Reichweite der Beta-Strahlung wird gleichzeitig das umgebende Ge-

Urol. Prax. 2020 - 22:100-104 https://doi.org/10.1007/s41973-020-00104-z

(c) Der/die Autor(en) 2020

\section{Wetterauer $\cdot$ H. Seifert $\cdot$ A. Chirindel · G. Nicolas \\ Die Rolle der PSMA-PET-CT in der Diagnostik und Therapie des Prostatakarzinoms}

\section{Zusammenfassung}

Die PSMA-PET-CT (ProstataspezifischesMembranantigen-Positronenemissionstomographie-Computertomographie) ist eine nuklearmedizinische Hybridbildgebung aus Schnittbilddiagnostik mittels CT (Computertomographie) und radioaktiv markiertem Tracer $\left({ }^{68} \mathrm{Gallium}\right.$ - oder ${ }^{18}$ Fluor-markiertem PSMA-Liganden). Die simultane Bildgebung kombiniert die Vorteile der molekularen und morphologischen Bildgebung und ist dadurch die derzeit sensitivste und spezifischste Methode zum Nachweis von Prostatakarzinomrezidiven. Die PSMA-PET-CT zeigt auch in der Primärdiagnostik und beim Staging für Karzinome mit mittlerem und hohem Risikoprofil eine höhere diagnostische Genauigkeit als die bisher verwendete konventionelle Bildgebung (CT und Knochenszintigraphie). Die PSMA-PET-CT ist auch ein obligatorisches bildgebendes Verfahren für die Selektion von Patienten, für welche die PSMA-gerichtete Radionuklidtherapie (mit ${ }^{177}$ Lutetium-markierten PSMA-Liganden) in Frage kommt. Diese neue zielgerichtete Therapie stellt eine vielversprechende Therapieoption in der Zweit- oder Drittlinientherapie bei Patienten mit metastasiertem und kastrationsrefraktärem Prostatakarzinom dar.

Schlüsselwörter

PSMA-PET-CT · Prostatakarzinom · PSARezidiv - Theragnostik · PSMA-Liganden

\section{Rôle de la TEP/TDM au PSMA dans le diagnostic et le traitement du cancer prostatique}

\section{Résumé}

La TEP/TDM au PSMA (tomographie par émission de positons/tomodensitométrie à l'antigène membranaire spécifique de la prostate) est une technique d'imagerie hybride alliant l'imagerie médicale en coupes par TDM et l'utilisation d'un traceur radiomarqué (ligand de PSMA marqué au ${ }^{68}$ gallium ou au ${ }^{18}$ fluor). L'imagerie simultanée combine les avantages de l'imagerie moléculaire et de l'imagerie morphologique, et constitue ainsi la méthode actuellement la plus sensible et la plus spécifique pour détecter les récidives de cancer de la prostate. La TEP/TDM au PSMA montre aussi une plus grande précision pour le diagnostic primaire et la stadification des carcinomes à profil de risque moyen et élevé que l'imagerie conventionnelle utilisée jusque-là (TDM et scintigraphie osseuse). La TEP/TDM au PSMA est de plus un procédé d'imagerie obligatoire pour la sélection des patients éligibles pour un traitement par radionucléides ciblant le PSMA (avec des ligands de PSMA marqués au ${ }^{177}$ lutétium). Ce nouveau traitement ciblé constitue une option prometteuse comme traitement de deuxième ou de troisième ligne chez les patients souffrant d'un cancer prostatique métastatique et réfractaire à la castration.

\section{Mots clés}

TEP/TDM au PSMA - Cancer de la prostate . Récidive biochimique (PSA) · Méthode théragnostique · Ligands du PSMA webe geschont. Die Erfahrungen der PSMA-gerichteten Radionuklidtherapie sind aussichtsreich. Bei mehr als der Hälfte der Patienten kann eine $50 \%$ ige Reduktion des PSA-Werts erreicht werden. Rund ein Drittel der Patienten spricht sehr gut auf die Therapie an, ein Drittel bleibt stabil. Ein weiteres Drittel spricht allerdings nicht auf die Therapie an [23, 24]. Die Nebenwirkungen beschränkten sich auf eine milde Knochenmarkstoxizität sowie gelegentliche
Mundtrockenheit. Risikoorgane sind vor allem die Speichel- und Tränendrüsen sowie das blutbildende Knochenmark. Die Wirksamkeit sowie das Toxizitätsprofil der PSMA-gerichteten Radionuklidtherapie sind vielversprechend und werden gegenwärtig in der Schweiz im Rahmen einer Register-Studie (wie z. B. am Universitätsspital Basel) und auch weltweit in einer randomisierten PhaseIII-Studie (Vision) weiter untersucht. 


\section{Fazit für die Praxis}

Die Prostatakarzinomdiagnostik konnte durch die Entwicklung des PSMALiganden deutlich verbessert werden. Da die meisten Prostatatumoren PSMA exprimieren, ist eine gute Darstellung von PSMA-exprimierendem Prostatakrebsgewebe und Metastasen mittels PSMA-PET-CT möglich. Bei unklaren Befunden sollte eine Biopsie erfolgen, zumal PSMA-exprimierende Zweittumoren selten sind. Die Ergebnisse der Radionuklidtherapie in der Drittlinientherapie des metastasierten und therapierefraktären Prostatakarzinoms sind vielversprechend.

\section{Korrespondenzadresse

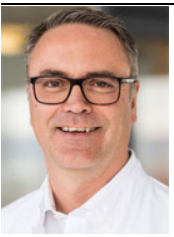 \\ Prof. Dr. Helge Seifert \\ Klinik für Urologie, Universitätsspital Basel Spitalstrasse 21, 4031 Basel Schweiz helge.seifert@usb.ch}

Funding. Open access funding provided by University of Basel

\section{Einhaltung ethischer Richtlinien}

Interessenkonflikt. C. Wetterauer, H. Seifert, A. Chirindel und $G$. Nicolas geben an, dass kein Interessenkonflikt besteht.

Für diesen Beitrag wurden von den Autoren keine Studien an Menschen oder Tieren durchgeführt. Für die aufgeführten Studien gelten die jeweils dort angegebenen ethischen Richtlinien.

Open Access. Dieser Artikel wird unter der Creative Commons Namensnennung 4.0 International Lizenz veröffentlicht, welche die Nutzung, Vervielfältigung, Bearbeitung, Verbreitung und Wiedergabe in jeglichem Medium und Format erlaubt, sofern Sie den/die ursprünglichen Autor(en) und die Quelle ordnungsgemäß nennen, einen Link zur Creative Commons Lizenz beifügen und angeben, ob Änderungen vorgenommen wurden.

Die in diesem Artikel enthaltenen Bilder und sonstige Drittmaterial unterliegen ebenfalls der genannten Creative Commons Lizenz, sofern sich aus der Abbildungslegende nichts anderes ergibt. Sofern das betreffende Material nicht unter der genannten Creative Commons Lizenz steht und die betreffende Handlung nicht nach gesetzlichen Vorschriften erlaubt ist, ist für die oben aufgeführten Weiterverwendungen des Materials die Einwilligung des jeweiligen Rechteinhabers einzuholen.
Weitere Details zur Lizenz entnehmen Sie bitte der Lizenzinformation auf http://creativecommons.org/ licenses/by/4.0/deed.de.

\section{Literatur}

1. Statistik. Bfr. (2020) Krebs in der Schweiz. https:// www.bfs.admin.ch/bfs/de/home/statistiken/ gesundheit.assetdetail.12107866.html. Zugegriffen: 6. Apr. 2020

2. Statistik Bf (2020) Neuerkrankungen und Sterbefälle nach Kreblokalisation, 2012-2016. https:// www.bfs.admin.ch/bfs/de/home/statistiken/ gesundheit/gesundheitszustand/krankheiten/ krebs/spezifische.assetdetail.12107886.html.Zugegriffen:6. Apr. 2020

3. Baratto L, Duan H, Macke H, lagaru A (2020) Imaging the distribution of gastrin-releasing peptidereceptors incancer.J Nucl Med 61:792-798

4. Pomper MG, Musachio JL, Zhang J, Scheffel U, Zhou Y, Hilton J et al (2002) 11C-MCG: synthesis, uptake selectivity, and primate PET of a probe for glutamate carboxypeptidase II (NAALADase). Mol Imaging 1:96-101

5. Conway RE, Petrovic N, Li Z, Heston W, Wu D, Shapiro LH (2006) Prostate-specific membrane antigen regulates angiogenesis by modulating integrin signal transduction. Mol Cell Biol 26:5310-5324

6. Hoffmann MA, Wieler HJ, Baues C, Kuntz NJ, Richardsen I, Schreckenberger M (2019) The impact of 68Ga-PSMA PET/CT and PET/MRI on the management of prostate cancer. Urology 130:1-12

7. Maurer T, Gschwend JE, Rauscher I, Souvatzoglou M, Haller B, Weirich Get al (2016) Diagnostic efficacy of (68)gallium-PSMA positron emission tomography compared to conventional imaging for lymph node staging of 130 consecutive patients with intermediate to high risk prostate cancer. JUrol 195:1436-1443

8. Eiber M, Weirich G, Holzapfel K, Souvatzoglou M, Haller B, Rauscher I et al (2016) Simultaneous (68)Ga-PSMA HBED-CC PET/MRI improves the localization of primary prostate cancer. Eur Urol 70:829-836

9. Afshar-Oromieh A, Haberkorn U, Eder M, Eisenhut M, Zechmann CM (2012) [68Ga]Galliumlabelled PSMA ligand as superior PET tracer for the diagnosis of prostate cancer: comparison with $18 \mathrm{~F}$ FECH. Eur J Nucl Med Mol Imaging 39:1085-1086

10. Shikanov S, Kocherginsky M, Shalhav AL, Eggener SE (2012) Cause-specific mortality following radical prostatectomy. Prostate Cancer Prostatic Dis 15:106-110

11. Afshar-Oromieh A, Alberts I, Sachpekidis C, Rominger A (2019) Prostate-specific membrane antigen (PSMA)-based diagnostics and treatment of prostate cancer. Urologe A 58:1429-1434

12. Perera M, Papa N, Christidis D, Wetherell D, Hofman MS, Murphy DG et al (2016) Sensitivity, specificity, and predictors of positive (68)Gaprostate-specific membrane antigen positron emission tomography in advanced prostate cancer: a systematic review and meta-analysis. Eur Urol 70:926-937

13. Perera M, Papa N, Roberts $M$, Williams $M$ Udovicich C, Vela I et al (2020) Gallium-68 prostate-specific membrane antigen positron emission tomography in advanced prostate cancer-updated diagnostic utility, sensitivity, specificity, and distribution of prostate-specific membrane antigen-avid lesions: a systematic review and meta-analysis. Eur Urol 77:403-417
14. Eiber $M$, Maurer $T$, Souvatzoglou $M$, Beer AJ, Ruffani A, Haller B et al (2015) Evaluation of hybrid (6)(8)Ga-PSMA Ligand PET/CT in 248 patients with biochemical recurrence after radical prostatectomy. J Nucl Med 56:668-674

15. Herlemann A, Wenter V, Kretschmer A, Thierfelder KM, Bartenstein P, Faber C et al (2016) (68)GaPSMA positron emission tomography/computed tomography provides accurate staging of lymph node regions prior to lymph node dissection in patients with prostate cancer. Eur Urol 70:553-557

16. Sheikhbahaei S, Afshar-Oromieh A, Eiber M, Solnes LB, Javadi MS, Ross AE et al (2017) Pearls and pitfalls in clinical interpretation of prostate-specific membrane antigen (PSMA)-targeted PET imaging. Eur J Nucl Med Mol Imaging 44:2117-2136

17. Afshar-Oromieh A, Debus $N$, Uhrig M, Hope TA, Evans MJ, Holland-Letz T et al (2018) Impact of long-term androgen deprivation therapy on PSMA ligand $\mathrm{PET} / \mathrm{CT}$ in patients with castration-sensitive prostate cancer. Eur J Nucl Med Mol Imaging 45:2045-2054

18. Emmett L, Yin C, Crumbaker M, Hruby G, Kneebone A, Epstein R et al (2019) Rapid modulation of PSMA expression by androgen deprivation: serial (68)Ga-PSMA-11 PET in men with hormone-sensitive and castrate-resistant prostate cancer commencing androgen blockade. JNucl Med 60:950-954

19. Mottet NBJ, Briers E, Bolla M, Bourke L, Cornford P, De Santis M, Henry A, Joniau S, Lam T, Mason MD, Van den Poel $\mathrm{H}$, Van den Kwast $\mathrm{TH}$, Rouvière $\mathrm{O}$ Wiegel T (2020) EAU - ESTRO - ESUR - SIOG guidelines on prostate cancer. EAU Annual Congress, Amsterdam. EAU Guidelines Office, Arnhem

20. Hofman MS, Lawrentschuk N, Francis RJ, Tang C, Vela I, Thomas Petal (2020) Prostate-specificmembrane antigen PET-CT in patients with high-risk prostate cancer before curative-intent surgery or radiotherapy (proPSMA): a prospective, randomised, multicentre study. Lancet 395:1208-1216

21. Budaus L, Leyh-Bannurah SR, Salomon G, MichI U, HeinzerH, Huland Hetal (2016) Initial experience of (68)Ga-PSMAPET/CT imaging in high-risk prostate cancer patients prior to radical prostatectomy. Eur Urol 69:393-396

22. Pyka T, Okamoto S, Dahlbender M, Tauber R, Retz M, Heck M et al (2016) Comparison of bone scintigraphy and (68)Ga-PSMA PET for skeleta staging in prostate cancer. Eur J Nucl Med Mol Imaging 43:2114-2121

23. Rahbar K, Ahmadzadehfar $\mathrm{H}$, Kratochwil $\mathrm{C}$, Haberkorn U, Schafers M, Essler M et al (2017) German multicenter study investigating 177LuPSMA-617 radioligand therapy in advanced prostate cancer patients. J Nucl Med 58:85-90

24. Hofman MS, Violet J, Hicks RJ, Ferdinandus J, Thang SP, Akhurst T et al (2018) [(177)Lu]-PSMA 617 radionuclide treatment in patients with metastatic castration-resistant prostate cance (LuPSMA trial): a single-centre, single-arm, phase 2 study. Lancet Oncol 19:825-833

Hinweis des Verlags. Der Verlag bleibt in Hinblick auf geografische Zuordnungen und Gebietsbezeichnungen in veröffentlichten Karten und Institutsadressen neutral. 
Hier steht eine Anzeige.

\section{曾 Springer}

